\title{
Interventions required in food system for better health outcome
}

\begin{abstract}
Overview
Food and agriculture are playing key roles for many of the global challenges such as poverty, under-nutrition, obesity, climate change, and biodiversity loss which impacts the health of the population of the country thus also impacts the economy of both developed and developing countries differently. Many changes are already implemented from food production to consumptions from various sectors but the efforts are still not enough and in infancy. The food system is a complicated network of tasks involving various steps from production, to consumption. The food system includes other factors of sociological factors like governance, the economics of food production, culture, health, sustainability, and its effect on the natural environment. This research paper will propose the primary changes required in the food system, policies, and dietary perspective to construct an integrated methodology of intervention, after evaluating web of interconnected different indicator variable and analyzing the previous methods of intervention responsible for food sustainability directly and indirect, at two different levels local and global. I will address the root cause of a problem in food system which impacts the health which includes various sectors such as socially, economically and politically channel at various levels in the food system to help the United Nations' Sustainable Development Goals (SDGs). Apart from this, the study also evaluates the economical burden from health issues resulting from the failing food system in developed and developing countries.
\end{abstract}

Keywords: food system, production, culture, health, sustainability
Volume I I Issue I - 202 I

\section{Ruchi Singh}

Health counselor \& Nutritionist, Long Island University, USA

Correspondence: Ruchi Singh, Health counselor \&

Nutritionist, Long Island University, USA,

Email ruchi5au@gmail.com

Received: February 15, 202I | Published: February 26, 202 I

\section{Introduction}

Many interrelated factors play role in food system sustainability which hinders the mission of the UN. We see a sharp contrast in developed countries and developing countries regard to the food system and related health outcomes. In contrast, we see malnutrition and under-nutrition in developing countries while in developed countries we see metabolic disorders due to obesity and overweight. Advancement in food commercialization and accelerated rate of fast food consumption triggered the involvement of giant food manufacturing companies to get higher profit and longer engagement from consumers, these food cooperation started using chemical stabilizers, taste stimulator and for example "Monosodium glutamate" to make people addicted to eating. With a tag of "zero calories," these big companies are using artificial sweeteners and hiding the deadly serious health consequences of its side effect.

The advanced techniques of agriculture and the use of GMO seeds and chemicals also adversely affected the climate. Food systems differ from country to country and, urban to rural areas. But what is the same everywhere in the? The impact of globalization also experiences in agricultural and rural areas of developing which has significant implication for food insecurity, environment change, and food sustainability.

To date many interventions, methods, policies from various sectors are established and implemented but still, they are far from solving the issue, on the opposite side, the healthcare economic burden on countries raised sharply due to chemical loaded, cheap and easy accessible packed food which created havoc on health and resulted into modern disease and at the same time quality food or fresh food are expensive, perishable and not easily accessible. This article emphasizes the urgent need for policies and practices to provide healthy food to growing population and at the same reduces the harm to the environment As per the article (there are two difficulties to accomplishing sustainable healthy food regimens for the world population, i.e., decrease in the production and nutritional quality of crop (specifically vegetables and organic products) because of environmental change; and compromises between food production and mechanical harvests.

As per the journal ${ }^{2}$ also The US food framework work inside an unpredictable nexus of social, political, monetary, social, and environmental elements. Among them are numerous powerful pressing factors, for example, population growth, urbanization, financial imbalances, environment pollution, and the growing interest for asset concentrated nutrients that place great strains on general wellbeing and the climate. This audit centers on the job that approach plays in characterizing the food framework, especially concerning agriculture. It further looks at the difficulties of making the food supply protected, nutritious, and maintainable while regarding the privileges, everything being equal, to approach satisfactory food and to accomplish the best quality of wellbeing. This study reason that the current US food framework is to a great extent undesirable, discriminatory, environment harming, and inadequately tough to persevere through the effects of environmental change, resource exhaustion, and population growth, and is thusly unreasonable. It is essential to transform the policies to change the food framework to support public health and the environment.

As per the article, ${ }^{3}$ low quality food habits are progressively prompting the trading off of human health leads to under nutrition and malnutrition in under privilege area while obesity, overweight along with other related co-morbidities are also. The worldwide energy for such activity is expanding, however, the proof base is expected to stir governments and consider partners responsible for remaining parts yet in infancy. Until now, contributions from researchers to this change plan have been ineffective, particularly given the interesting commitments the field can make as far as thorough examination and specialized counsel. The reinforced investment will require 
developments in measurements and approaches, joined with a new deduction on what comprises feasible proof and a more prominent ability to draw in with private area agri-food actors.

Many studies suggested that intricate field, customary exploration, and strategy that connects across different areas are barely aligned and is limited and commonly neglects to accept conceivably key hidden changes in the more extensive food framework, and this study needs new methods instead of conventional ones. The literature journals and exploration networks that have advanced around the food framework and healthcare and food framework and climate plans over the previous many years need to merge and combine, explaining compromises, and looking for co-benefits and synergistic arrangements. Lacks in food system prompts prevent the objective of food maintainability which study ${ }^{4}$ believe to incorporate factors such as climate, culture, and different components.

Many variables in the food system from various dimensions lead to hinder the goal of food sustainability. Most of these variables which I think are local (like cultural, religious) to that place, and addressing the local factors which include environment, culture and other factors are very important for the sustainable food system. I think we should categorize the issue into two categories, Generalizable issue and Local issue.

Literature review: For the literature review, categorizing the issues of the sustainable food system in three but interrelated heading will be easy to progress in research.

\section{Deficiencies in food system}

As per the article ${ }^{5}$ current system is not capable of food sustainability goal and in fact the hunger, under nutrition and obesity are increasing and stated the problem in insufficient supply, inefficient deliver, and unequal access, unaffordable and unsustainable

As per the article "Repairing food systems failures: Policies, Innovations \& Partnerships" by Ruben the basic need to keep moving of food frameworks disappointments gets obvious in five related issues:

I. Insufficient supply of food from agriculture to take care of to feed the growing population.

II. Inefficient transportation of food from farmers to consumers because of wasteful agro-coordination and wastage of food during transport, storage.

III. Unequitable excess to food market for healthy and nutritious food, because of highly sectioned/segmented food markets,

IV. Unaffordable opportunity for poor people groups' utilization of better food sources, since better nutrient rich food are expensive than ultra processed food.

V. Unsustainable food supply because of negative natural effects as far as land and water use, biodiversity and energy force of ozone harming substance discharges.

According to Ruben ${ }^{5}$ fundamental elements for a more strategic food systems approach need to include attention for five mutually related FOCUS areas:

a. Focusing on structural constraints for improving access and availability of foods, particularly opportunities for increasing supply chain efficiency and reducing the costs of healthier diets,

b. Optimizing the institutional design of agri-food value chains, considering the perishable nature of many fresh foods and reducing the risks of quality degradation and food losses,

c. Combining technological innovations with behavioral change, including effective incentives for "nudging" consumers and producers towards safer and healthier foods,

d. Upgrading the full array of public sector agents (agro-logistics; food safety) and private sector stakeholders (traders, processors, retailers) into joint programs of food-business innovation.

e. Supporting dual interventions that strengthen both the food environment (supply side) as well as activities that influence consumer food choice and preferences (demand-side).

The study by $\mathrm{y}^{6,7}$ emphasizes that receiving a practical food framework plan suggests acknowledgment of its political economy measurement, recognizing that numerous cultural entertainers have tasks to carry out at various levels in getting sorted out food frameworks and making them more economical and more impartial. Not just neighborhood and public governments are to be included but private area actors and common society associations also. To guarantee a productive and constructive exchange between various leaders the multifaceted nature of sustainable food frameworks and the requirement for tending to compromises and interaction should be explained and recognized by all. As per the report by ${ }^{8}$ There is an urgent need for evidence-based policies and programs and investment in resources to solve the challenging issues related to food sustainability into addressing the most basic difficulties of our occasions: alleviating environmental change through changes to food creation and utilization, and guaranteeing that individuals internationally approach nutritious, reasonable and earth manageable food. These issues are not only basic for wellbeing and the climate but also for the worldwide economy, trade and jobs. Environmentfriendly agriculture; motivations for the consumer to change their diet; nutrient food and innovative technologies combined with thorough sociology are crucial for tending to the underlying drivers of our failing worldwide food framework.

The investigation by ${ }^{9}$ has distinguished the factors that impact agriculture production, food security, and foreign exchange. After finding the regressor and regress and regression analysis, the study suggests that the supportability of the food supply in Russia is undermined by inflation and decreasing purchasing power of the population force them to buy cheaper quality products and the same time exporters seeking higher profit from foreign countries creating a shortage in the local market. The worldwide patterns of diets and the food frameworks that produce those diet plans recommend that they are neither nutritious nor sustainable, which has suggestions for accomplishing SDG2. This paper ${ }^{10}$ portrays the present status of the worldwide eating pattern and food system, and ethical concerns in achieving the same. As per the article, ${ }^{11}$ high expensive food cost due to agriculture intensifications can be reduced by using natural resources more carefully more efficiently, and sustainably, for instance using targeted fertilizers carefully and natural weather which also helps to reduce the use of energy and minerals thus lowering carbon emission, reduce environmental impacts and achieve multiple benefits. Reorienting agriculture dependent on near favorable circumstances in agricultural production, reducing entry boundaries into the metropolitan labor market and up-skilling the rural and metropolitan labor force are keys to creating an evenhanded food system for the future. ${ }^{12}$

The most important and prime goal of the food system is health. ${ }^{13}$ All the Advances in the health sciences keep on fortifying the rule that 
nutritious food is fundamental for the accomplishment of full physical and mental potential for all people and for supporting wellbeing through the aging process. Moreover, advances in the social and behavioral sciences are uncovering the numerous elements of health, the practices that promote health, which create more productive agricultural frameworks and maintainable turn of events. It is additionally an arising power in the farming approach, driven partially by the rise of the "triple weight" of malnutrition - the concurrence of hunger, nutrition inadequacies, and overweight and obesity, due to excessive calorie intake because of an absence of concordance between food frameworks and health.

This article ${ }^{14}$ contends that EU administration structures stay badly adjusted to the fundamental idea of this and different difficulties in food frameworks (for example environmental change, biodiversity loss, food insecurity): clashing goals and missed collaborations are distinguished between various approach regions (agricultural, health, environment and so forth) and between various degrees of governance (national, local). A coordinated food system - a 'Common Food Policy' - is accordingly needed to meet the EU's general health and manageability goals. It distinguishes four unique parts of the administration move needed to advance health and construct a sustainable food system in Europe: (I) cognizance across policies; (ii) coordination across governance levels; (iii) administration for transition; and (iv) food democracy. Outlines for a Common Food Policy are as of now arising and are ready for thought, improvement, and usage by the European foundations.

\section{Faulty food and healthcare policies}

As per the article by ${ }^{5}$ changes in food policies that are basic for lessening craving and hunger are:

a) Substantial interests in agro-coordination to control the expenses of transportation and reduce the waste in the agro-food chain,

b) Shifting the concentration from agrarian (area) policies to food policies, with more focus on consumer demand performance criteria.

c) Focusing on strategy coherence focusing on health and climate profits by interests in improved food frameworks (as determined by IFPRI: 1 US $\$$ interest in better food may reduce healthcare costs by 16 US\$)

d) Leapfrogging on specialized advancement in data and correspondence innovations (ICT) supporting home conveyance and customized nourishment applications for distraught shoppers,

e) Upgrading the well-being and nature of street-foods, out-ofhome utilization, and handled food sources to control the fast development of the overweight problem.

f) Focusing on new collusions between new policies and business practice offers partnerships that are imperative structure blocks for quickening the joint challenge of arriving at Zero Hunger (SDG2).

g) Some other ways for dealing with these challenges are as suggested by (siani.se/news-story/our-food-systems-are-failingus-how-can-we-turn-this-around, 2020). ${ }^{15}$

h) Reroute cultivating and country livelihoods by reducing ozonedepleting substance outflows, diminishing disparities, improving social incorporation, and strengthening while at the same time advancing environment tough rural practices that address dietary issues. i) De-risk livelihood, farms, and worth chains with an early warning system for natural calamities utilizing the advanced information and communication system

j) Reduce emission from diets and value chains with an emphasis on changing the dietary pattern to reduce food loss and waste. Changing how supply chains, food retail, advertising, and the acquisition would all be able to help accomplish these objectives.

k) Realign approaches, money, backing to social developments, and advancement to fabricate stronger and reasonable food frameworks. This is the foundation of the three other activity territories, empowering approaches and establishments should ensure they are realigning appropriations and exchange, managing power disparities and underestimation just as supporting social developments that work with environment, livelihoods, and food framework issues.

The variables like attitude perceived behavioral control, subjective norms, economic, sensory, cultural factors are important determinants of sustainable food consumption as well as behavior which can be used for further empirical studies. ${ }^{16}$ The article by ${ }^{17}$ outline the advancement, difficulties, and health benefit of adopting a food frameworks strategy that unites the food, agribusiness, and general wellbeing fields.

\section{Deficiency in both policies and food system}

This paper $^{18}$ investigates different difficulties and opportunities to accomplish nutrition sustainability in the future. They unite the most recent data accessible on the advancement process in India to (1) feature the idea of food framework challenges, (2) recognize patterns and threats to advance, (3) stress policies and institutional interventions to address difficulties, and (4) set a plan interlinking agriculture, health and nutrition; and economic advancement.

\section{Methods/solutions}

As per the article in. ${ }^{19}$ Commission proposes five methodologies as broad beginning stages for national, regional, city, and local change

1. Seek global and public obligation to move toward a healthy diet. Shifting to a planetary wellbeing diet will require global use of food sources, for example, red meat and sugar to reduce by half, while the use of organic products, nuts, vegetables, and vegetables should be double. Policies are needed to improve the availability, access, and affordability of healthy foods.

2. Reorient rural needs from delivering a high quantity of food to producing healthy food which also needs new policies.

3. Sustainably strengthen food creation to produce high-quality food. Use innovation and framework development to cultivate the existing area

4. Strong and facilitated governance of land and seas. Protect environments and biodiversity by acting, at local and global levels, to stop the extension of agricultural land and marine zones.

5. At least split food loss and waste, following UN Sustainable Development Goals.

This study ${ }^{20}$ utilize a modified adaptation of Donella Meadows' thought of 'leverage points' - places in complex frameworks where moderately little changes can prompt conceivably extraordinary foundational changes. Study outcomes demonstrate that the kind of intercessions concentrated in the literature are incompletely determined 
by research techniques and issue framings and that 'profound influence focuses' identified with changing the framework's rules, values, and standards are rarely addressed. the study recommends that for sustainable framework wide groundbreaking change, deep leverage points - the goals of a system, its intent, and rules - need to be addressed more directly and it requires explicit consideration of how scientific approaches shape and constrain our understanding of where we can intervene in complex systems.

The study ${ }^{21}$ found that the dietary regime of Swiss society of nutrient is the most manageable choice and is projected to bring about 36\% lesser environmental footprint, 33\% lesser expenditure and $2.67 \%$ lower adverse health outcome (DALYs) contrasted and the current eating routine. On the other hand, high animal food consumption lead adverse health outcome, natural impression, day by day food use and a decrease in the intake of an essential nutrient ( Vitamin C, Fiber, Potassium, and Calcium).study found that moving to vegetarian diet situations may lead to a decrease in the intake of specific micronutrients (Vitamin B12, Choline, and Calcium). Results show that accomplishing a reasonable diet shift would involve a decrease in the intake of meat and vegetable oils and a moderate decrease in grains, roots, and fish items, and simultaneously expanded admission of vegetables, nuts, seeds, and leafy foods. We distinguish a few flow information and examination holes that should be taken care of in request to get more exact outcomes. This study underscores the need to think about numerous indicators while evaluating dietary sustainability and gives a template to direct such investigations in different nations and settings. Future endeavors should zero in on surveying the capability of various intercessions and approaches that can help progress the populace from current to reasonable dietary examples.

This review ${ }^{22}$ found that life-cycle based approaches are not only useful for identifying hotspots for impact reduction, but also for comparing the performance of different sustainability interventions. In particular, interventions targeting the production phase, such as promoting dietary change through menu planning in which highimpact ingredients (e.g., animal products) are replaced with low-impact ingredients (e.g., plant foods), had the highest improvement potential. Interventions targeting other phases of the catering supply chain (e.g., food storage, meal preparation, waste management) had considerably lower improvement potentials. This review article provides valuable insights on how the sustainability of the foodservice sector can be improved without the burden-shifting of impacts, which interventions to priorities, and where knowledge gaps in research exist. A key recommendation for future research is to focus on combined life cycle thinking approaches that are capable of addressing sustainability holistically in the foodservice sector by integrating and assessing the environmental, social, and economic dimensions of interventions.

This study ${ }^{23}$ was unable to discover any proof concerning if the digital behavior change interventions inspected worked. Most investigations further needed hypothetical establishing to deal with how or why they ought to be compelling for conduct change for more sustainable food consumption practices. This study suggests future examination in the field is to extend from the current exploratory stage to conduct the scientifically rigorous study and investigations of more quality, all the more completely grounded in conduct change hypothesis and strategies. Besides, given the study, they propose changes to the Behavior Change Technique Taxonomy.

Refocusing policies from disease control to prevention will improve worldwide coordination endeavors in pandemic counteraction. Executing such proactive activities will cost little from budget and this should also include the economic loss due to pandemics. ${ }^{24}$ This policy brief ${ }^{25}$ was set up on the side of the Austrian EU Presidency to investigate how the food system can join diet-related health with ecological and financial arrangement objectives. It expands on extensive prior work by breaking down the associations between various strategic objectives and between strategic objectives and food frameworks. Through this interaction, the creators distinguish 3 center parts of food frameworks working which would have to interface (economic benefits for farmers and businesses derived from the production and delivery of nutritious food using sustainable methods) to produce co-benefits.

This study $^{7}$ recognizes four distinct pathways for food framework change and blueprint their insightful underpinnings, their perspectives on multi-stakeholder governance, and how they manage basic compromises between various food framework goals. Studies suggest that food framework approaches should be valuable to policymakers, and execution must be improved if leaders have a superior comprehension of these hidden connections and elements of food system change.

The current work by the InteAcademy Partnership (IAP), ${ }^{26}$ the worldwide organization of science institutes, unites set up provincial organizations of foundations, shaping another coordinated effort focused on with this present institutes' program is to bring the science force of foundations to zero in on the 16 extended food, nutrients $t$ and agribusiness issues. Another commitment is the accentuation on food frameworks and in that setting a critical accentuation on strength of individuals and the climate. (IAP, 2018). Suggestions include: universally supporting and sharing essential and applied examination for improved food, nutrients, and agriculture.

Interpretation of exploration to advancement requires more grounded associations across disciplines and with forefront advances, linkage to science education, training and effort, and Upgrading logical foundation (Food Systems - Definition, Concept, and Application for the UN Food Systems Summit, 2020). This policy brief mentioned action tracks, cross-cutting issues and brief, and stated objective of the sustainable food system. Aims of the policy brief given below:

a. Creating practical food and sustenance frameworks, taking a frameworks viewpoint to convey health and well being, connected to change towards the roundabout economy and bio-economy.

b. Underscoring change to a healthy diet and good nutrition.

c. Understanding food production and use issues, covering contemplations of productivity, supportability, environmental dangers, and variety of assets.

d. Exploiting openings going in close vicinity to go in the biosciences and other quickly propelling sciences.

e. Tending to the food-energy-supplements water-wellbeing nexus, perceiving that limits are obscured.

f. Advancing movement at the science-strategy interfaces and accommodating arrangement disengages.

g. Combining and organizing worldwide science warning systems.

The work by ${ }^{4}$ modeling research recommends that yields of significant cereals will decay under situations of increased temperature, particularly in tropical nations and agricultural land is increasing in the clash with infrastructure development and with protected zones. Biodiversity loss and loss of soil quality will both significantly affect worldwide foods grown from the ground supply 
and consequently on the health of the population. Ongoing spikes in the cost of food is already a concern in almost 20 nations. There are many chances to moderate or decrease these effects that will turn out to be progressively significant as the interest in food keep on rising in a socio-economical, societal, and political context.

After discussing different great practices and food strategy conversations comparable to the change of current food frameworks toward their social, natural, and efficient manageability. The papers ${ }^{27}$ portray strategies, projects, and activities in creating and progressed economies of Europe and Central Asia that refer to the center components of food frameworks, for example, food supply, food environment, and consumer. The shared insights, research, analysis, studies and approaches, encounters, and experiences add to a better comprehension of regional specificities and supporting the endeavors to guide the complex food frameworks' transformation to deliver a healthy and nutrient rich diet.

This article ${ }^{28}$ gives an outline of the key effects produced by current food frameworks on human and ecological health, with emphasis on their effect in the area of Europe and Central Asia. This study proofs how differentiated agro-biological frameworks can take a vital part in health and nutrient objectives, while additionally addressing the natural, social, and monetary challenges made by current frameworks. Eventually, the article reveals insight into loop wholes and recommends how more enhanced frameworks can successfully be advanced through foundational, multi-area, and integrated policies to deal with challenges and ensure a healthy diet.

\section{Adverse health outcome}

The study by. ${ }^{29}$ Discovers proof for substantial development in the kinds and amounts of UPFs (ultra-processed food) sold around the world, addressing a change towards a more handled global eating pattern yet with wide varieties among areas and nations. In richer nations, higher volumes and a more extensive assortment of UPFs are sold. Trends are most noteworthy in Australasia, North America, Europe, and Latin America however filling quickly in Asia, the Middle East, and Africa. These advancements are firmly connected with the industrialization of food frameworks, innovative change, and globalization, including development for the market and political involvement of transnational food cooperation and weak, policies to provide nutrients. This dietary pattern progress, especially in populated middle-income nations, raises genuine health concerns globally. As per the news $\mathrm{in}^{30}$ costs of health care in the U.S. often focus on the high costs of treating diseases, rather than on the potential economic benefits of preventing them. America spends more than any other country other but still, the cases of chronic cases keep on rising. Preventing approach and creating awareness among the population is the best way to address the cost drivers.

As the aging population rising ${ }^{31}$ the food security and chronic conditions are common among the elderly, they observed higher health care costs in the presence of this interaction for those who were food insecure and had poor health than for those who were food secure. As per the journal article ${ }^{32}$ in 2017, 11 million disability-adjusted lifeyears (DALYs) attributed to dietary factors globally in many countries. This study showed the picture that many non-communicable diseases related to dietary. This paper ${ }^{33}$ recommended actions to prevent noncommunicable disease among low- and middle-income countries for government, policymakers to take essential steps of subsidizing the cost of vegetable, fruits, and grains, develop policies for health diet and system, public officials should create education programs, the healthcare system must equip to deal with a non-communicable disease, create a system to tackle both problems of obesity and malnutrition and researcher should focus in changing diet and supply factors. This ${ }^{34}$ study analyzed the relationship between an unhealthy diet and non-communicable disease (NCD) and cost burden due to NCD. This analysis insisted on the need of transforming the food system to tackle the problem of NCD and they recommended action to create a healthy food environment.

The panel report ${ }^{35}$ reviewed literature and concluded: "Reliable proof showed a dietary pattern that is higher in plant-based food sources, for example, vegetables, natural products, entire grains, vegetables, nuts, and seeds, and lower in animal-based food, is more health and wellbeing promoting and is related with lesser ecological effect (ozone harming substance outflows and energy, land, and water use) than is the current average U.S. diet.

There is an urgent requirement for additional engagement and commitment in the policies, practice, and research exercises expected to propel food frameworks and general wellbeing approach. The strategy openings portrayed. ${ }^{17}$ three among numerous different models - show the incredible guarantee for mutually improving health, food, agriculture, climate, and the economy. A per the document on the website, ${ }^{36}$ Agriculture records for most of antimicrobial use in the United States, with around $70 \%$ of therapeutically significant antitoxins sold cross country being utilized in this area. Antimicrobial obstruction in the climate is a huge general health concern and full comprehension of the effect of low degrees of anti-toxins in the climate. Normal agriculture practices add to general food framework weakness. Pesticide use upholds mono-cropping practice increase crop weakness, and pesticides hurt natural life and useful insects, for example, pollinators that are necessary for food production. The use of chemical damages the soil $\mathrm{pH}$ which affect the soil quality. Extreme climate changes connected to environmental change, for example, heatwaves, dry season, and draught, sway the quality and quantity and food distribution channels. Research also showed a high level of carbon dioxide which adversely impacts the nutrient level. This Healthcare Perspectives article ${ }^{37}$ surveys recent distributions on current mediations usually utilized in the food business, including hereditary designing, pesticides, anti-microbial, chemicals, and food added substances, and recorded their adverse consequences for the human body. It likewise talks about the advantages of avoiding industrial food sources and choose locally grown organic food to prevent disease and get nutritious food.

\section{Indicators for sustainable development goals}

The Sustainable Development Goals (SDG) indicators that have been identified by ${ }^{38}$ and agreed upon so far, and those that are still in process, will probably contribute towards a better way of measuring and communicating food security results in the years to come. Such communication will need to include the social, economic, and environmental sustainability of the global food system(s) that food security is based upon to fully address all four pillars of food security namely availability, access, utilization, and stability. To what degree the good news or the bad news narrative will dominate future food security communication is still to be seen, but the chances are that both will continue and mutually challenge each other. This study also showed the weakness of the indicators for the correct measurement. As per the study, the result of research such as CGIAR's, attribution is difficult to assess, as so many actors are involved in the process, from knowledge and technology to usage and food security improvements. Not everything that counts can be counted, hence, operating quantifiable food security indicators in combination with anecdotal evidence could be one way of providing a 
better understanding of food security results. Governing the world by numbers has its limitations, but governing the world without numbers would probably be even more problematic.

The website small footprint family ${ }^{39}$ suggested 13 ways to attain a sustainable food system by giving examples. The report from ZERO HUNGER CHALLENGE WORKING GROUPS (agencies, 2015) found the key determinants of the food system and proposed the principles for a sustainable food system which include Interdisciplinary thinking, Consensus building, Mapping and assessment, interconnected decision-making, Evidence-based, Continuous learning. The paper ${ }^{40}$ used the example of the local tomato supply chain to demonstrate livelihood narrative favors the environment narrative and local supply chain create a positive environment for the farmer. This study has exhibited that the investigation of food supply chains can be semantically outlined from alternate points of view, which involves distinctive issue organizing in proper terms and various exhibitions. Indeed, each chain performs uniquely in contrast to the viewpoint of every account: Thusly, the utilization of traits and markers from various measurements and scales has served to distinguish. The more challenging in any event, while generating quantitative outcomes utilizing a perspective- - e.g., economic - the subsequent indicator can be seen in various manners by various actors.

The article by ${ }^{41}$ recognized the ten forces that shaping the global food system, these are degradation of natural resources, climate change, culture and tradition, urbanization, globalization, consumer behavior, technology innovation, sustainability, government policies, conflict, and fragile states. Many proofs confirm that environmental change, extreme climate, and decreasing natural resources address significant difficulties for the food system to satisfy growing population need both economically meet human sustenance needs and follow planetary imperatives. ${ }^{42}$ The food framework measurements study precisely measure food framework execution across all applicable spaces of interest: nutrient, climate, financial, social, versatility, security, and waste. This new procedure accordingly permits measurement of supportable sustenance security (SNS), a methodology which would now be able to be used by decision-makers and investors to set significant objectives, track progress on SDGs, and assess the expected effect of food framework intercessions planned to improve both human and planetary wellbeing. As demonstrated by in study models, the measurements can be applied to both developed and less developed countries. in this paper, the measurements applied at the public scale, but it can also able applied at the geographic scale with, but with the prerequisite for gathering and revealing information of better spatial solution. This ${ }^{42}$ study also noticed that an absence of lack policies in some areas highlighted the area for action.

In this review paper, ${ }^{43}$ existing food and nutrition security indicators, discuss some of their advantages and disadvantages, and finally, classify them and describe their relationships and overlaps. To achieve this, the paper refers to the existing definitions of food and nutrition security (FNS), in particular as they have been agreed upon and implemented in the Food Secure project (www.food-secure. EU). The main existing conceptual frameworks of FNS predating the present paper are also used as guidelines and briefly discussed. This study provides recommendations for food security and concluded the study with five elements explaining usability and non-usability of various indicators.

The results of the study ${ }^{44}$ showed that sustainable development (SD) changed from different periods that is before the 1972 embryonic period, (1972-1987) modeling period, (1987-present). SD is gradually evolving from targeting just a single goal of sustainable resources to millennium development goals and sustainable goals and culture, good governance, and life support are important in pursuing SD. This paper finds ${ }^{45}$ and argues that the entire issue of sustainable development centers around inter-and intra-generational equity anchored essentially on three-dimensional distinct but interconnected pillars, namely the environment, economy, and society. Decision-makers need to be constantly mindful of the relationships, complementarities, and tradeoffs among these pillars and ensure responsible human behavior and actions at the international, national, community, and individual levels to uphold and promote the tenets of this paradigm in the interest of human development.

It advocates ${ }^{46}$ viewing sustainable development not only as the establishment of a permanent, globally practicable, and future-capable mode of life and economics, but as a complex array of problems involving a wide range of social-scientific and humanistic disciplines. This innovative approach means that the book is oriented toward current problems, not toward the established academic boundaries, and it draws out lessons that are relevant for those studying and working in sustainability across the world. ${ }^{47}$

\section{Conclusion}

After review of many journals, textbooks, blogs, and reports from different integrated sectors of the food system which include -policies, governance, food system structure, and food habits, I can sum of the actions needed for sustainable food system research in the below points:

A. Strengthening the food system by improving supply chain efficiency, food availability and reducing the costs of healthier diets.

B. Renovating the agri-food chain to avoid food loss and quality degradation during transportation.

C. Utilization of the innovative method of agriculture to preserve the environment's natural resources and at the same time preventing the atmosphere from pollution.

D. Integrating the awareness program among people to change food habit with food supplier so that then they can mutually get benefited

E. Public and private sectors stakeholder need to work together for creating effective food, health, and economic policies based on evidence.

F. Bringing together leaders from different sectors to advocate the sustainable food system.

G. Integration, cooperation, and coordination of officials from different levels of government are required for reaching the goal.

H. Establishing the organization for checking the impacts of ultrarefined food and various preservatives on the general public and its negative outcome.

\section{Possible methodological limitations}

It is possible that I missed some of the important journal for reviewing. I think more research is needed from the economic and socio structure point. Unavailability of data on some interrelated issue the impacts the conclusion of the study. More research is needed to gather data. 


\section{Acknowledgments}

None.

\section{Conflicts of interest}

The author declares that there are no conflicts of interest.

\section{Funding}

None.

\section{References}

1. Lindgren E, Harris F, Alan D, et al. Sustainable food systems - a health perspective. Sustainability Science. 2018;1505-1517.

2. Shannon KL, Kim BF, McKenzie SE. Food system policy, public health, and human rights in the United States. Annu Rev Public Health. 2015.

3. Lartey A, Meerman J, Wijesinha-Bettoni R.. Why Food System Transformation Is Essential and How Nutrition Scientists Can Contribute. Annals of Nutrition and Metabolism. 2018

4. Dangour AA, Mace G, Shankar B. Food systems, nutrition, health and the environment. The Lancet Planetary Health. 2017.

5. Ruben R. Repairing food systems failures: Policies, Innovations \& Partnerships.

6. Béné C, Oosterveer P, Lamotte L, et al. When food systems meet sustainability - Current narratives and implications for actions. World Development. 2019;16-13.

7. Brouwer ID, McDermott J, Ruben R. Food systems everywhere: Improving relevance in practice. Global Food Security. 2020

8. Hurley-Depret M. Global food systems are failing humanity and speeding up climate change. Washington, DC; 2018.

9. Erokhin V. Factors Influencing Food Markets in Developing. MDPI. 2017.

10. Fanzo J. Healthy and Sustainable Diets and Food Systems: the Key to Achieving Sustainable Development Goal 2? Food Ethics. 2019;159174.

11. Environmental impacts of food systems. 2016.

12. Pingali P. Economic Growth, Agriculture and Food Systems: Explaining Regional Diversity. Transforming Food Systems for a Rising India. 2019;15-45.

13. The food system and its interaction with human health and nutrition (2020). International food policy research Institute. Washington, DC.

14. Schutter OD, Jacobs N, Clément C. A 'Common Food Policy' for Europe: How governance reforms can spark a shift to healthy diets and sustainable food systems. Food Policy. 2020.

15. siani.se/news-story/our-food-systems-are-failing-us-how-can-we-turnthis-around. 2020.

16. Ifeanyichukwu $\mathrm{CD}$, Nwaizugbo IC. Exploring Critical Factors Influencing Sustainable Food. European Journal of Business and Innovation Research. 2020.

17. Neff RA, Merrigan K, Wallinga D. A Food Systems Approach To Healthy Food And Agriculture Policy. Health Affairs. 2015.

18. Pingali P, Aiyar A, Abraham M, et al. Indian Food Systems towards 2050: Challenges and Opportunities. Transforming Food Systems for a Rising India. 2019.

19. www.hsph.harvard.edu/nutritionsource/sustainability

20. Dorninger C, Abson DJ, Apetrei CI. Leverage points for sustainability transformation: a review on interventions in food and energy systems. Ecological Economics. 2020.

21. Chen C, Chaudhary A, Mathys A. Dietary Change Scenarios and Implications for Environmental, Nutrition, Human Health and Economic Dimensions of Food Sustainability. MDPI. 2019.

22. Takacs B, Borrion A. The Use of Life Cycle-Based Approaches in the Food Service Sector to Improve Sustainability: A Systematic Review. MDPI. 2020.

23. Hedin B, Katzeff C, Eriksson E, et al. A Systematic Review of Digital Behaviour Change Interventions for More Sustainable Food Consumption. MDPI. 2019.

24. Aiya A, Pingali P. Pandemics and food systems - towards a proactive food safety approach to disease prevention \& management. Food Security. 2020

25. Kelly Parsons $\mathrm{CH}$. Connecting food systems for co-benefits: How can food syste combnine diet related health with environmental and economy policy goal. Copenhagen: European Observatory on Health Systems and Policies. 2018.

26. Braun JV, Afsana K, Fresco L, et al. Food Systems - Definition, Concept and Application for the UN Food Systems Summit. 2021

27. Dupouy E, GurinovicM. Sustainable food systems for healthy diets in Europe and Central Asia: Introduction to the special issue. Food Policy. 2020 .

28. Frison E, Clément $\mathrm{C}$. The potential of diversified agroecological systems to deliver healthy outcomes: Making the link between agriculture, food systems \& health. Food Policy. 2020.

29. Baker P, Machado P, Santos T, et al. Ultra-processed foods and the nutrition transition: Global, regional and national trends, food systems transformations and political economy drivers. Public Health/Nutrition. 2020

30. Waters MH, Graf M. Chronic diseases are taxing our health care system and our economy. 2018.

31. Garcia SP, Haddix A, Barnett K.. Incremental Health Care Costs Associated With Food Insecurity and Chronic Conditions Among Older Adults. Preventing Chronic Disease, public health research. 2018.

32. Collaborators G. Health effects of dietary risks in 195 countries, 1990 2017: a systematic analysis for the Global Burden of Disease Study 2017. The lancet. 2019.

33. Bankman J. Chronic Disease,Changing Diets, and Sustainability. New York: Brighter Green. 2017.

34. Branca F, Lartey A, Oenema S, et al. Transforming the food system to fight non-communicable diseases. BMJ. 2019;364.

35. Millen BE, Abrams S, Adams-Campbell L, et al. The 2015 Dietary Guidelines Advisory Committee Scientific Report: Development and Major Conclusions. Advances in Nutrition. 2016.

36. Community health risks of industrial agriculture. 2018.

37. Hong H. Modern Industrial Foods and Their Effects on the Human Body. Natural Medicine Journal. 2016.

38. Haug R. Food security indicators:How to measure and communicate results. Norwegian University of Life Sciences. 2018.

39. Gifford D. 3 Ways to Create a Sustainable Food System. 2021.

40. Gamboa G, Kovacic Z, Masso MD, et al. The Complexity of Food Systems: Defining Relevant. Spain: MDPI. 2016

41. Denning G, Fanzo J. Ten Forces Shaping the Global Food System. 2021.

42. Gustafson D, Gutman A, Leet W, et al. Seven Food System Metrics of Sustainable. MDPI. 2016. 
43. Pangaribowo Hanie E., Gerber N., Torero Maximo. Food and nutrition security indicators. Econstor. 2013.

44. Shi L, Han L, Yang F, et al. The Evolution of Sustainable Development Theory. MDPI. 2019;11(24):7458.

45. Mensah J, Casadevall SR. Sustainable development: Meaning, history, principles, pillars, and implications for human action: Literature review. Cognet social science. 2019.
46. Enders JC, Remig M. Theories of Sustainable Development. Routledge. 2016

47. All Food Systems Are Sustainable. Compendium -Final Report Zero Hunger Challenge Working Groups. 2015. 